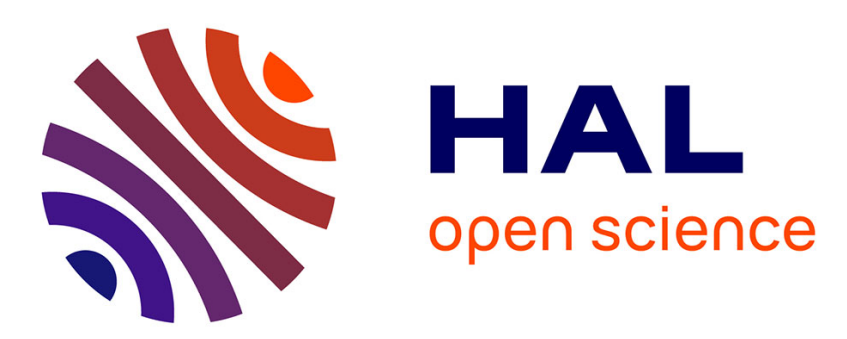

\title{
Entanglement-assisted quantum parameter estimation from a noisy qubit pair: A Fisher information analysis \\ François Chapeau-Blondeau
}

\section{To cite this version:}

François Chapeau-Blondeau. Entanglement-assisted quantum parameter estimation from a noisy qubit pair: A Fisher information analysis. Physics Letters A, 2017, 381 (16), pp.1369-1378. 10.1016/j.physleta.2017.02.037 . hal-02171134

\section{HAL Id: hal-02171134 \\ https://hal.science/hal-02171134}

Submitted on 22 Jun 2021

HAL is a multi-disciplinary open access archive for the deposit and dissemination of scientific research documents, whether they are published or not. The documents may come from teaching and research institutions in France or abroad, or from public or private research centers.
L'archive ouverte pluridisciplinaire HAL, est destinée au dépôt et à la diffusion de documents scientifiques de niveau recherche, publiés ou non, émanant des établissements d'enseignement et de recherche français ou étrangers, des laboratoires publics ou privés. 


\title{
Entanglement-assisted quantum parameter estimation from a noisy qubit pair: A Fisher information analysis
}

\author{
François Chapeau-Blondeau
}

\author{
Laboratoire Angevin de Recherche en Ingénierie des Systèmes (LARIS), Université d'Angers, 62 avenue Notre Dame du Lac, 49000 Angers, France
}

\begin{abstract}
Benefit from entanglement in quantum parameter estimation in the presence of noise or decoherence is investigated, with the quantum Fisher information to asses the performance. When an input probe experiences any (noisy) transformation introducing the parameter dependence, the performance is always maximized by a pure probe. As a generic estimation task, for estimating the phase of a unitary transformation on a qubit affected by depolarizing noise, the optimal separable probe and its performance are characterized as a function of the level of noise. By entangling qubits in pairs, enhancements of performance over that of the optimal separable probe are quantified, in various settings of the entangled pair. In particular, in the presence of the noise, enhancement over the performance of the one-qubit optimal probe can always be obtained with a second entangled qubit although never interacting with the process to be estimated. Also, enhancement over the performance of the two-qubit optimal separable probe can always be achieved by a two-qubit entangled probe, either partially or maximally entangled depending on the level of the depolarizing noise.
\end{abstract}

\section{Introduction}

For extracting information from measurement at a quantum level, an important task is quantum parameter estimation. For quantum parameter estimation, typically one has access to a quantum system in a state $\rho_{\xi}$ dependent on the unknown parameter $\xi$ to be estimated. One chooses a measurement protocol applied to the quantum system in state $\rho_{\xi}$, and the measurement outcomes are processed by means of an estimator $\widehat{\xi}$ in order to infer a value for the parameter $\xi$. From classical estimation theory [1] it is known that any conceivable estimator $\widehat{\xi}$ for $\xi$ is endowed with a mean-squared error $\left\langle(\widehat{\xi}-\xi)^{2}\right\rangle$ which is lower bounded by the Cramér-Rao bound involving the reciprocal of the classical Fisher information $F_{c}(\xi)$. Estimators are known, such as the maximum likelihood estimator, that reach the Cramér-Rao bound in definite (usually asymptotic) conditions. Higher Fisher information $F_{c}(\xi)$ generally entails higher performance in estimation, and one has then the faculty to select the quantum measurement protocol so as to maximize $F_{c}(\xi)$. In this respect, there is a fundamental upper bound $\left[2-4\right.$ ] provided by the quantum Fisher information $F_{q}\left(\rho_{\xi} ; \xi\right)$ which sets a limit to the classical Fisher information $F_{c}(\xi)$, imposing $F_{c}(\xi) \leq F_{q}\left(\rho_{\xi} ; \xi\right)$. In turn, constructive methodologies (usually adaptive) exist [5-10] yielding a measurement protocol reaching

E-mail address: chapeau@univ-angers.fr.
$F_{c}(\xi)=F_{q}\left(\rho_{\xi} ; \xi\right)$. The quantum Fisher information $F_{q}\left(\rho_{\xi} ; \xi\right)$ has thus the status of a relevant metric characterizing the ultimate best performance achievable in quantum parameter estimation based on a quantum state $\rho_{\xi}$, and we shall use it as such in this paper.

A definite measurement operating on a single copy of the quantum system in state $\rho_{\xi}$ can be repeated $N$ times on $N$ independent copies of the quantum system prepared in the same state $\rho_{\xi}$. In this situation of independent state preparation and independent measurement, the quantum Fisher information, alike the classical Fisher information, is additive [11] and amounts to $N F_{q}\left(\rho_{\xi} ; \xi\right)$. This is associated with a mean-squared error $\left\langle(\widehat{\xi}-\xi)^{2}\right\rangle$ decreasing as $1 / N$, forming the standard limit or shot-noise limit of the error $[12,13]$. This $1 / N$ scaling of the mean-squared error remains true when a joint measurement is performed collectively on the $N$ copies of the quantum system when prepared independently [12, 13]. Such preparation corresponds for the $N$-fold quantum system to a joint state $\rho_{\xi}^{(N)}=\rho_{\xi}^{\otimes N}$ which is separable. Quantum physics through the unique property of entanglement offers an alternative for improved estimation. When the $N$-fold quantum system is prepared in a joint state $\rho_{\xi}^{(N)}$ which is not separable but entangled, then through collective, or even separable, measurement a mean-squared error scaling as $1 / N^{2}$ may be accessible, forming the quantum enhancement or Heisenberg limit of the error [14, $15,12]$. This is a specifically quantum benefit, since classically, introducing correlation among $N$ measurements is generally unable to yield an estimation performance better than the $1 / N$ scaling; 
by contrast, quantum correlation under the form of entanglement is able to improve the estimation performance from $1 / N$ to $1 / N^{2}$ scaling of the mean-squared error.

Such quantum enhancement however is not accessible for any parametric dependence [11]. It is also fragile to quantum noise or decoherence. Quantum estimation, initiated in noise-free conditions, has more recently been addressed in the presence of noise or decoherence $[16,13,17,18]$. Especially, in the asymptotic limit of large $N$, it has been shown that any small amount of depolarizing noise is sufficient to ruin the $1 / N^{2}$ performance and return it to $1 / N[11,13,19]$. In addition, the asymptotic condition of large $N$, although it stands as an important theoretical reference, may be difficult to handle in practice since it involves preparing, controlling and measuring very large entangled states which remain, with the current quantum technologies, very uneasy tasks. By contrast, the simplest form of quantum entanglement, most robust to decoherence and easiest to handle analytically and practically, starts to become accessible with a qubit pair. It is therefore realistic and practically relevant to seek to make the most of quantum entanglement at the level of qubit pairs. This is the direction we follow in the sequel, where we concentrate on analyzing benefit for estimation that can arise from entanglement in a qubit pair in the presence of quantum noise. We address the generic situation of estimation of the phase angle of a unitary transformation acting on a qubit in the presence of quantum depolarizing noise, with the quantum Fisher information to assess the performance. We investigate the benefit for estimation of exploiting a qubit pair in various entanglement settings. Several specific situations have been analyzed of entanglement-assisted schemes for estimation and metrology, with overviews provided in [15] with no noise, and more recently in [13] with noise. However, relatively few studies derived explicit expressions for the quantum Fisher information $F_{q}\left(\rho_{\xi} ; \xi\right)$ in meaningful situations in the presence of noise, because this is usually recognized as a difficult task. Here, we identify and work out meaningful situations where such derivations are feasible, and provide in this way additional quantitative characterizations for entanglement-assisted estimation in the presence of quantum noise.

\section{Quantum Fisher information}

In this section, we recall an expression accessible for the quantum Fisher information of a general quantum state and that will be useful to us in the sequel. In addition, we establish a convexity property over a class of transformed quantum states and relevant to our study.

To proceed with the estimation task introduced in Section 1, we consider a quantum system in a $D$-dimensional Hilbert space $\mathcal{H}_{D}$ having its state represented by the density operator $\rho_{\xi}$ dependent upon an unknown parameter $\xi$. For estimating $\xi$ by measuring $\rho_{\xi}$, the ultimate best performance is controlled by the quantum Fisher information $F_{q}\left(\rho_{\xi} ; \xi\right)$ contained in the density operator $\rho_{\xi}$ about the parameter $\xi$. By referring to the spectral decomposition of $\rho_{\xi}$ in its orthonormal eigenbasis $\rho_{\xi}=\sum_{n=1}^{D} \lambda_{n}\left|\lambda_{n}\right\rangle\left\langle\lambda_{n}\right|$, one has access to the expression [20-22]

$F_{q}\left(\rho_{\xi} ; \xi\right)=2 \sum_{m, n} \frac{\left|\left\langle\lambda_{m}\left|\partial_{\xi} \rho_{\xi}\right| \lambda_{n}\right\rangle\right|^{2}}{\lambda_{m}+\lambda_{n}}$,

where the sums as in Eq. (1) include all terms corresponding to eigenvalues $\lambda_{m}+\lambda_{n} \neq 0$. For the special case of a pure state $\rho_{\xi}=|\lambda\rangle\langle\lambda|$ then the derivative $\partial_{\xi} \rho_{\xi}=\left|\partial_{\xi} \lambda\right\rangle\langle\lambda|+| \lambda\rangle\left\langle\partial_{\xi} \lambda\right|$, and the expression of Eq. (1) should be replaced $[21,22]$ by

$F_{q}\left(\rho_{\xi} ; \xi\right)=4\left(\left\langle\partial_{\xi} \lambda \mid \partial_{\xi} \lambda\right\rangle+\left\langle\partial_{\xi} \lambda \mid \lambda\right\rangle^{2}\right)$.
In general, the quantum Fisher information $F_{q}\left(\rho_{\xi} ; \xi\right)$ when seen as a functional of the density operator $\rho_{\xi}$ is a convex $(U)$ functional of $\rho_{\xi}[23,24]$, in the sense that if $\rho_{\xi}$ is a convex combination of density operators, such as $\rho_{\xi}=p^{\prime} \rho_{\xi}^{\prime}+p^{\prime \prime} \rho_{\xi}^{\prime \prime}$, then $F_{q}\left(\rho_{\xi} ; \xi\right) \leq p^{\prime} F_{q}\left(\rho_{\xi}^{\prime} ; \xi\right)+p^{\prime \prime} F_{q}\left(\rho_{\xi}^{\prime \prime} ; \xi\right)$. As a consequence, when there is no constraint on $\rho_{\xi}$, then $F_{q}\left(\rho_{\xi} ; \xi\right)$ is maximized by a density operator $\rho_{\xi}$ which is not convexly decomposable into some other density operators, i.e. a rank-one density operator or pure state of the form $\rho_{\xi}=|\psi\rangle\langle\psi|$.

In many circumstances, comprising the ones we investigate here, the $\xi$-dependent quantum state $\rho_{\xi}$ is constrained to be of a form realized from a $\xi$-independent quantum state $\rho_{0}$ (an input probe) which experiences transformation by a $\xi$-dependent quantum process denoted $\mathcal{T}_{\xi}(\cdot)$ to yield $\rho_{\xi}=\mathcal{T}_{\xi}\left(\rho_{0}\right)$. As an arbitrary transformation of a quantum state, quantum theory [25] imposes that $\mathcal{T}_{\xi}(\cdot)$ is a completely positive trace-preserving linear map. The map $\mathcal{T}_{\xi}(\cdot)$ can represent a unitary evolution of a quantum state, or also a nonunitary evolution involving decoherence. As a consequence of the linearity of the map $\rho_{\xi}=\mathcal{T}_{\xi}\left(\rho_{0}\right)$, the quantum Fisher information $F_{q}\left(\rho_{\xi}=\mathcal{T}_{\xi}\left(\rho_{0}\right) ; \xi\right)$ when seen as a functional of the input density operator $\rho_{0}$, is also a convex $(U)$ functional of $\rho_{0}$. When $\rho_{0}$ is a convex combination of density operators, such as $\rho_{0}=p_{0}^{\prime} \rho_{0}^{\prime}+p_{0}^{\prime \prime} \rho_{0}^{\prime \prime}$, then by linearity $\mathcal{T}_{\xi}\left(\rho_{0}\right)=p_{0}^{\prime} \mathcal{T}_{\xi}\left(\rho_{0}^{\prime}\right)+p_{0}^{\prime \prime} \mathcal{T}_{\xi}\left(\rho_{0}^{\prime \prime}\right)$, and by the convexity in $\rho_{\xi}$ therefore

$F_{q}\left(\mathcal{T}_{\xi}\left(\rho_{0}\right) ; \xi\right) \leq p_{0}^{\prime} F_{q}\left(\mathcal{T}_{\xi}\left(\rho_{0}^{\prime}\right) ; \xi\right)+p_{0}^{\prime \prime} F_{q}\left(\mathcal{T}_{\xi}\left(\rho_{0}^{\prime \prime}\right) ; \xi\right)$

expressing convexity in $\rho_{0}$.

Equation (3) implies that if the input probe $\rho_{0}$ can be convexly decomposed under a form $\rho_{0}=p_{0}^{\prime} \rho_{0}^{\prime}+p_{0}^{\prime \prime} \rho_{0}^{\prime \prime}$, then $F_{q}\left(\rho_{\xi}=\right.$ $\left.\mathcal{T}_{\xi}\left(\rho_{0}\right) ; \xi\right)$ is not maximal at this $\rho_{0}$. Conversely, $F_{q}\left(\rho_{\xi}=\mathcal{T}_{\xi}\left(\rho_{0}\right) ; \xi\right)$ is at a maximum necessarily for a $\rho_{0}$ that is not convexly decomposable. The states $\rho_{0}$ that are not convexly decomposable are rank-one density operators of the form $\rho_{0}=\left|\psi_{0}\right\rangle\left\langle\psi_{0}\right|$. This establishes the important property that the quantum Fisher information $F_{q}\left(\rho_{\xi}=\mathcal{T}_{\xi}\left(\rho_{0}\right) ; \xi\right)$ accessible from the transformed state $\rho_{\xi}=\mathcal{T}_{\xi}\left(\rho_{0}\right)$ is maximized by a pure input probe $\rho_{0}=\left|\psi_{0}\right\rangle\left\langle\psi_{0}\right|$.

\section{Single qubit with noise}

When $\rho_{\xi}$ is a qubit state in the two-dimensional Hilbert space $\mathcal{H}_{2}$, a convenient parametrization is offered by the Bloch representation [25]

$\rho_{\xi}=\frac{1}{2}\left(\mathrm{I}_{2}+\vec{r}_{\xi} \cdot \vec{\sigma}\right)$

with $\mathrm{I}_{2}$ the identity of $\mathcal{H}_{2}$, and $\vec{\sigma}$ a formal vector assembling the three $2 \times 2$ (traceless Hermitian unitary) Pauli matrices $\left[\sigma_{x}, \sigma_{y}, \sigma_{z}\right]=\vec{\sigma}$. The coordinates of $\rho_{\xi}$ are specified by the Bloch vector $\vec{r}_{\xi}$ in $\mathbb{R}^{3}$, with norm $\left\|\vec{r}_{\xi}\right\|=1$ for a pure state and $\left\|\vec{r}_{\xi}\right\|<1$ for a mixed state. For the qubit in Bloch representation, the quantum Fisher information of Eq. (1) follows [22] as

$F_{q}\left(\rho_{\xi} ; \xi\right)=\frac{\left(\vec{r}_{\xi} \cdot \partial_{\xi} \vec{r}_{\xi}\right)^{2}}{1-\vec{r}_{\xi}^{2}}+\left(\partial_{\xi} \vec{r}_{\xi}\right)^{2}$

for a mixed state, and for the pure state of Eq. (2) as

$F_{q}\left(\rho_{\xi} ; \xi\right)=\left(\partial_{\xi} \vec{r}_{\xi}\right)^{2}$.

An important family of parametric qubit states $\rho_{\xi}$ arises [21, 22] when an initial qubit, forming the input probe, is prepared in an initial state $\rho_{0}$ and then subjected to a $\xi$-dependent unitary transformation $U_{\xi}$ of general form

$\mathrm{U}_{\xi}=\exp \left(-i \frac{\xi}{2} \vec{n} \cdot \vec{\sigma}\right)$ 
where $\vec{n}=\left[n_{x}, n_{y}, n_{z}\right]^{\top}$ is a real unit vector of $\mathbb{R}^{3}$, so as to deliver the transformed state $\rho_{\xi}=\mathrm{U}_{\xi} \rho_{0} \mathrm{U}_{\xi}^{\dagger}$. In Bloch representation, the unitary transformation of Eq. (7) implements in $\mathbb{R}^{3}$ a rotation by the angle $\xi$ around the axis $\vec{n}$ of the initial Bloch vector $\vec{r}_{0}$ of the input probe $\rho_{0}$ into the transformed Bloch vector $\vec{r}_{\xi}$. In such circumstance, the quantum Fisher information of both Eqs. (5) and (6) evaluates [26] to $F_{q}\left(\rho_{\xi} ; \xi\right)=\left(\vec{n} \times \vec{r}_{0}\right)^{2}$, uniformly for any $\xi$. Maximization of this $F_{q}\left(\rho_{\xi} ; \xi\right)$ is therefore achieved by an optimal input probe $\rho_{0}$ in a pure state (as already known from Section 2) characterized by a unit Bloch vector $\vec{r}_{0}$ orthogonal to the rotation axis $\vec{n}$, this to reach the overall maximum $F_{q}\left(\rho_{\xi} ; \xi\right)=F_{q}^{\max }=1$. This defines the conditions for the ultimate best performance in estimating the phase shift $\xi$ inflicted to a qubit by a unitary transformation.

The above situation stands as the important reference of a noise-free or decoherence-free qubit. An arguably more realistic situation would be when the transformed qubit state $\mathrm{U}_{\xi} \rho_{0} \mathrm{U}_{\xi}^{\dagger}$, before it becomes accessible to measurement for estimating $\xi$, is affected by a quantum noise or by decoherence, as we now consider. On the transformed qubit state now denoted $\rho_{1}(\xi)=\mathrm{U}_{\xi} \rho_{0} \mathrm{U}_{\xi}^{\dagger}$, the action of a quantum noise or of decoherence can always be expressed as a completely positive trace-preserving linear map under the operator-sum representation [25]

$\rho_{1}(\xi) \longrightarrow \mathcal{N}\left(\rho_{1}\right)=\sum_{\ell} \Lambda_{\ell} \rho_{1}(\xi) \Lambda_{\ell}^{\dagger}=\rho_{\xi}$,

with the Kraus operators $\Lambda_{\ell}$ satisfying $\sum_{\ell} \Lambda_{\ell}^{\dagger} \Lambda_{\ell}=\mathrm{I}_{2}$.

When estimation of the phase angle $\xi$ has to be performed from the noisy qubit state $\rho_{\xi}$ resulting from Eq. (8), then the optimization of the input probe $\rho_{0}$ and the maximum it affords for the Fisher information $F_{q}\left(\rho_{\xi} ; \xi\right)$ of Eqs. (5)-(6), are usually critically dependent on the noise $\mathcal{N}(\cdot)$ in Eq. (8) and also on the parameter $\xi$ itself. Such optimal conditions have been explicitly worked out in [22] for different quantum noise models relevant to the qubit. This dependence of the optimal probe $\rho_{0}$ on the unknown parameter $\xi$ usually entails the practical complication of the necessity of resorting to adaptive iterative estimation protocols in order to approach maximal performance, as for instance performed in [5-10]. There is however an important situation where such complication can be avoided. This is the situation of a noise of great relevance to the qubit, which is the depolarizing noise.

The depolarizing noise $[25,27]$ implements a quantum operation $\mathcal{N}(\cdot)$ in Eq. (8) taking the form

$\mathcal{N}\left(\rho_{1}\right)=(1-p) \rho_{1}+\frac{p}{3}\left(\sigma_{x} \rho_{1} \sigma_{x}^{\dagger}+\sigma_{y} \rho_{1} \sigma_{y}^{\dagger}+\sigma_{z} \rho_{1} \sigma_{z}^{\dagger}\right)$

where the action of the noise is to leave the qubit state $\rho_{1}(\xi)$ unchanged with the probability $1-p$ or to apply any one of the three Pauli operators with equal probability $p / 3$. Alternatively, the transformation of Eq. (9) can be put [25] under the equivalent (but non-Kraus) form $\mathcal{N}\left(\rho_{1}\right)=\alpha \rho_{1}+(1-\alpha) \mathrm{I}_{2} / 2$ with $\alpha=1-4 p / 3$, manifesting an isotropic geometrical character of the depolarizing noise, which in a probabilistic way either leaves the qubit state $\rho_{1}(\xi)$ unchanged or replaces it by the maximally mixed state $I_{2} / 2$. This isotropic character is also manifested in Bloch representation [25], where the action of the depolarizing noise of Eq. (9) is to apply to the qubit Bloch vector $\vec{r}_{1}(\xi)$ a uniform compression $\vec{r}_{1} \rightarrow \alpha \vec{r}_{1}=\vec{r}_{\xi}$ by the factor $\alpha=1-4 p / 3$ satisfying $0 \leq|\alpha| \leq 1$. This isotropic or highly symmetric character of the depolarizing noise makes it more tractable analytically; and also the depolarizing noise is somehow a worse-case scenario for quantum information [28], and in this respect it enables a conservative picture accessible as a tractable reference.
This isotropy of the depolarizing noise entails that the Fisher information of both Eqs. (5) and (6) evaluates [26] to $F_{q}\left(\rho_{\xi} ; \xi\right)=$ $\alpha^{2}\left(\vec{n} \times \vec{r}_{0}\right)^{2}$ uniformly for any $\xi$, and that $F_{q}\left(\rho_{\xi} ; \xi\right)$ is therefore maximized by a pure input probe $\rho_{0}$ (as already known from Section 2) characterized by a unit Bloch vector $\vec{r}_{0}$ orthogonal to the rotation axis $\vec{n}$ as in the noise-free case, this to achieve the maximum

$F_{q}\left(\rho_{\xi} ; \xi\right)=F_{q}^{\max }=\alpha^{2}$,

matching the noise free-case $F_{q}^{\max }=1$ when $p=0$ and $\alpha=1$.

When the above estimation scenario is repeated on $N$ independent qubits prepared in the same state $\rho_{0}$ with the noise acting independently on each qubit, then the quantum Fisher information is additive [11] and amounts to $N F_{q}\left(\rho_{\xi} ; \xi\right)$ experiencing a gain by $N$. By contrast, with $N$ entangled qubits, the gain in the quantum Fisher information may be raised at most to $N^{2}[12,11]$. However, not all types of dependence on $\xi$ can benefit from such quantum enhancement, and with large increasing $N$ the gain in Fisher information returns to $N$ instead of $N^{2}$ as soon as a small amount of depolarizing noise is present [11]. Moreover, as argued in the Introduction section, since large populations of entangled qubits are difficult to produce and control, it is therefore realistic to concentrate on small populations of entangled qubits, and examine the best benefit they afford for estimation in the presence of noise. In this direction, we study how the estimation scenario on one noisy qubit considered in this section and optimized to achieve the maximal quantum Fisher information of Eq. (10), can benefit from entanglement when it is worked out on a qubit pair.

\section{Pair of qubits with noise}

We now consider a qubit pair in $\mathcal{H}_{2}^{\otimes 2}$ which is prepared in the joint state denoted as before by $\rho_{0}$ and forming the input probe for our estimation task. The density operator $\rho_{0}$ is now a general two-qubit state, possibly entangled or separable, allowing to examine the impact thereof on the estimation performance.

For the sake of definiteness, we consider the unitary transformation

$\mathrm{U}_{\xi}=\left[\begin{array}{cc}1 & 0 \\ 0 & e^{i \xi}\end{array}\right]$.

Such $\mathrm{U}_{\xi}$ can be factored as $\mathrm{U}_{\xi}=e^{i \xi / 2} \exp \left(-i \xi \sigma_{z} / 2\right)$ which is equivalent to the unitary $\exp \left(-i \xi \sigma_{z} / 2\right)$ falling in the family of Eq. (7), since the prefactor $e^{i \xi / 2}$ cancels out and has no impact in the transformation $\rho \rightarrow \mathrm{U}_{\xi} \rho \mathrm{U}_{\xi}^{\dagger}$ of quantum states. As argued in [11], Eq. (11) is a generic unitary transformation, also considered in many other studies, which retains the essential features of a general transformation as in Eq. (7), where an arbitrary orientation like $\vec{n}$ in Eq. (7) is assimilated to the $O z$ axis ${ }^{1}$ with Eq. (11).

A unitary transformation $\mathrm{U}_{\xi}$ on a qubit density operator can be fully characterized by its action on the canonical basis $\{|j\rangle\langle k| ; j, k=0,1\}$ of operators on $\mathcal{H}_{2}$ onto $\mathcal{H}_{2}$, denoted $|j\rangle\left\langle k\left|\rightarrow U_{\xi}\right| j\right\rangle\langle k| U_{\xi}^{\dagger}=\mathcal{U}_{\xi}(|j\rangle\langle k|)$. With $U_{\xi}$ from Eq. (11), this yields the characterization

$$
\begin{aligned}
& \mathcal{U}_{\xi}(|0\rangle\langle 0|)=|0\rangle\langle 0|, \\
& \mathcal{U}_{\xi}(|0\rangle\langle 1|)=e^{-i \xi}|0\rangle\langle 1|, \\
& \mathcal{U}_{\xi}(|1\rangle\langle 0|)=e^{i \xi}|1\rangle\langle 0|, \\
& \mathcal{U}_{\xi}(|1\rangle\langle 1|)=|1\rangle\langle 1| .
\end{aligned}
$$

\footnotetext{
1 The $O z$ axis is the axis of $\mathbb{R}^{3}$ carrying the two (antipodal) Bloch vectors defining the two orthogonal eigenstates $|0\rangle$ and $|1\rangle$ of the Pauli operator $\sigma_{z}$. In this way $\exp \left(-i \xi \sigma_{z} / 2\right)$ represents a rotation in $\mathbb{R}^{3}$ of angle $\xi$ around $\vec{n}=[0,0,1]^{\top}$.
} 
In a similar way, the action of the depolarizing noise of Eq. (9) on a qubit density operator is fully characterized by the four transformations

$\mathcal{N}(|0\rangle\langle 0|)=\frac{1+\alpha}{2}|0\rangle\left\langle 0\left|+\frac{1-\alpha}{2}\right| 1\right\rangle\langle 1|$,

$\mathcal{N}(|0\rangle\langle 1|)=\alpha|0\rangle\langle 1|$,

$\mathcal{N}(|1\rangle\langle 0|)=\alpha|1\rangle\langle 0|$,

$\mathcal{N}(|1\rangle\langle 1|)=\frac{1-\alpha}{2}|0\rangle\left\langle 0\left|+\frac{1+\alpha}{2}\right| 1\right\rangle\langle 1|$,

with the noise factor $\alpha=1-4 p / 3 \in[-1 / 3,1]$.

Cascading the two above quantum operations $\mathcal{N} \circ \mathcal{U}_{\xi}(\cdot)=$ $\mathcal{N}\left[\mathcal{U}_{\xi}(\cdot)\right]=\mathcal{T}_{\xi}(\cdot)$ yields

$\mathcal{T}_{\xi}(|0\rangle\langle 0|)=\frac{1+\alpha}{2}|0\rangle\left\langle 0\left|+\frac{1-\alpha}{2}\right| 1\right\rangle\langle 1|$,

$\mathcal{T}_{\xi}(|0\rangle\langle 1|)=\alpha e^{-i \xi}|0\rangle\langle 1|$,

$\mathcal{T}_{\xi}(|1\rangle\langle 0|)=\alpha e^{i \xi}|1\rangle\langle 0|$,

$\mathcal{T}_{\xi}(|1\rangle\langle 1|)=\frac{1-\alpha}{2}|0\rangle\left\langle 0\left|+\frac{1+\alpha}{2}\right| 1\right\rangle\langle 1|$.

It can especially be verified that $\mathcal{N}(\cdot)$ and $\mathcal{U}_{\xi}(\cdot)$ commute on the basis states $\{|j\rangle\langle k| ; j, k=0,1\}$, so that everywhere $\mathcal{N} \circ \mathcal{U}_{\xi}(\cdot)=$ $\mathcal{U}_{\xi} \circ \mathcal{N}(\cdot)=\mathcal{T}_{\xi}(\cdot)$; therefore the unitary and the noise applied either way to a qubit have the same effect.

The probe state $\rho_{0}$ is a general two-qubit state that we refer to the canonical basis $\left\{\left|j j^{\prime}\right\rangle\left\langle k k^{\prime}|=| j\right\rangle\left\langle k|\otimes| j^{\prime}\right\rangle\left\langle k^{\prime}\right| ; j, j^{\prime}, k, k^{\prime}=0,1\right\}$ of operators on $\mathcal{H}_{2}^{\otimes 2}$ onto $\mathcal{H}_{2}^{\otimes 2}$, with a matrix representation of row index $\operatorname{bin}\left(j j^{\prime}\right)$ and column index bin $\left(k k^{\prime}\right)$, as $\rho_{0}=\left[\rho_{\text {bin }\left(j j^{\prime}\right) \text { bin }\left(k k^{\prime}\right)}\right]=$ $\rho_{00}|00\rangle\left\langle 00\left|+\rho_{01}\right| 00\right\rangle\left\langle 01\left|+\rho_{02}\right| 00\right\rangle\left\langle 10\left|+\ldots+\rho_{33}\right| 11\right\rangle\langle 11|$, where $\operatorname{bin}\left(j j^{\prime}\right)$ represents the integer value between 0 and 3 of the binary sequence $j j^{\prime}$.

\subsection{One qubit transformed}

We first examine the setting, depicted in Fig. 1, where only the first qubit of the pair experiences the unitary plus noise transformation, while the second qubit is left untouched. Comparable settings associating an active qubit entangled with an inactive qubit have already been considered for instance in [23,14,29-32, $13,33]$. The characterization we develop here is novel of the quantum Fisher information with depolarizing noise for such a qubit pair. For the setting of Fig. 1, the resulting two-qubit noisy transformed state is $\rho_{\xi}=\mathcal{T}_{\xi} \otimes \mathcal{I}\left(\rho_{0}\right)$, with $\mathcal{I}(\cdot)$ the one-qubit identity operation defined by $\mathcal{I}(|j\rangle\langle k|)=|j\rangle\langle k|$ over the canonical basis.

Using the characterization of Eqs. (20)-(23) for the one-qubit quantum operation $\mathcal{T}_{\xi}(\cdot)$ and performing the tensor product, one deduces for any input probe $\rho_{0}$, the two-qubit noisy transformed state $\rho_{\xi}=\mathcal{T}_{\xi} \otimes \mathcal{I}\left(\rho_{0}\right)$ under the matrix representation in the canonical basis,

$\rho_{\xi}=\left[\begin{array}{cccc}b \rho_{00}+c \rho_{22} & b \rho_{01}+c \rho_{23} & \alpha e^{-i \xi} \rho_{02} & \alpha e^{-i \xi} \rho_{03} \\ b \rho_{10}+c \rho_{32} & b \rho_{11}+c \rho_{33} & \alpha e^{-i \xi} \rho_{12} & \alpha e^{-i \xi} \rho_{13} \\ \alpha e^{i \xi} \rho_{20} & \alpha e^{i \xi} \rho_{21} & c \rho_{00}+b \rho_{22} & c \rho_{01}+b \rho_{23} \\ \alpha e^{i \xi} \rho_{30} & \alpha e^{i \xi} \rho_{31} & c \rho_{10}+b \rho_{32} & c \rho_{11}+b \rho_{33}\end{array}\right]$,

with the shorthand notation $b=(1+\alpha) / 2$ and $c=(1-\alpha) / 2$. Its derivative readily follows as

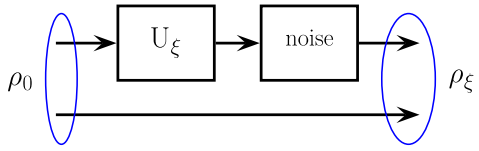

Fig. 1. A pair of qubits in a generally entangled state where only the first qubit of the pair experiences the unitary plus noise transformation, while the second qubit is left untouched.

$$
\partial_{\xi} \rho_{\xi}=\left[\begin{array}{cccc}
0 & 0 & -i \alpha e^{-i \xi} \rho_{02} & -i \alpha e^{-i \xi} \rho_{03} \\
0 & 0 & -i \alpha e^{-i \xi} \rho_{12} & -i \alpha e^{-i \xi} \rho_{13} \\
i \alpha e^{i \xi} \rho_{20} & i \alpha e^{i \xi} \rho_{21} & 0 & 0 \\
i \alpha e^{i \xi} \rho_{30} & i \alpha e^{i \xi} \rho_{31} & 0 & 0
\end{array}\right] .
$$

It is now feasible from Eqs. (24) and (25), to compute the quantum Fisher information $F_{q}\left(\rho_{\xi} ; \xi\right)$ of Eq. (1). This requires to perform the eigendecomposition $\left\{\lambda_{n},\left|\lambda_{n}\right\rangle\right\}$ of the $4 \times 4$ density matrix $\rho_{\xi}$ of Eq. (24), and then use the derivative $\partial_{\xi} \rho_{\xi}$ of Eq. (25) to evaluate its matrix elements $\left\langle\lambda_{m}\left|\partial_{\xi} \rho_{\xi}\right| \lambda_{n}\right\rangle$ and deduce $F_{q}\left(\rho_{\xi} ; \xi\right)$ of Eq. (1). For an arbitrary input probe $\rho_{0}$, with no special structure or symmetry (other than the built-in Hermiticity), this computation of $F_{q}\left(\rho_{\xi} ; \xi\right)$, although in principle analytically feasible, is however practically very cumbersome and can alternatively be accomplished numerically. There is nevertheless an interesting class of input states $\rho_{0}$ where this computation can be handled analytically to provide useful insight on benefit from entanglement for estimation.

This class is formed by the 2-qubit pure states in $\mathcal{H}_{2}^{\otimes 2}$ under the form

$\left|\psi_{0}\right\rangle=\sqrt{1-q}|0\rangle \otimes|0\rangle+\sqrt{q}|1\rangle \otimes|1\rangle=\sqrt{1-q}|00\rangle+\sqrt{q}|11\rangle$.

By varying the Schmidt coefficient $q$ between 0 and 1 , it is possible to span between a maximally entangled state at $q=1 / 2$ and two separable states at $q=0$ and $q=1$. Such a flexible class of entangled states has previously been exploited to explore the impact of entanglement in estimation. It is exploited for instance in [23], where the quantum Fisher information is also used so as to assess the estimation of a one-parameter non-unitary quantum channel, yet with no extra noise affecting the measured quantum state as we consider here. The characterization we develop here of the quantum Fisher information for estimating the phase of a unitary transformation with depolarizing noise is novel. From Eq. (26), the input probe follows with the density operator $\rho_{0}=\left|\psi_{0}\right\rangle\left\langle\psi_{0}\right|$ as

$$
\begin{aligned}
\rho_{0}= & (1-q)|00\rangle\langle 00|+\sqrt{(1-q) q}(|00\rangle\langle 11|+| 11\rangle\langle 00|) \\
& +q|11\rangle\langle 11|,
\end{aligned}
$$

having the matrix form

$\rho_{0}=\left[\begin{array}{cccc}1-q & 0 & 0 & \sqrt{(1-q) q} \\ 0 & 0 & 0 & 0 \\ 0 & 0 & 0 & 0 \\ \sqrt{(1-q) q} & 0 & 0 & q\end{array}\right]$

The resulting two-qubit noisy transformed state $\rho_{\xi}=\mathcal{T}_{\xi} \otimes$ $\mathcal{I}\left(\rho_{0}\right)$ from Eq. (24) is derived in Appendix A together with the ensuing quantum Fisher information $F_{q}\left(\rho_{\xi} ; \xi\right)$ of Eq. (1). The derivation of Appendix A shows a quantum Fisher information $F_{q}\left(\rho_{\xi} ; \xi\right)$ in Eq. (A.6) which is independent of the unknown parameter $\xi$, and which is maximized by the Schmidt coefficient $q=1 / 2$, i.e. for a maximally entangled pure state for the input probe $\rho_{0}$ from Eq. (26). The corresponding maximal Fisher information achieved in Eq. (A.6) follows as 


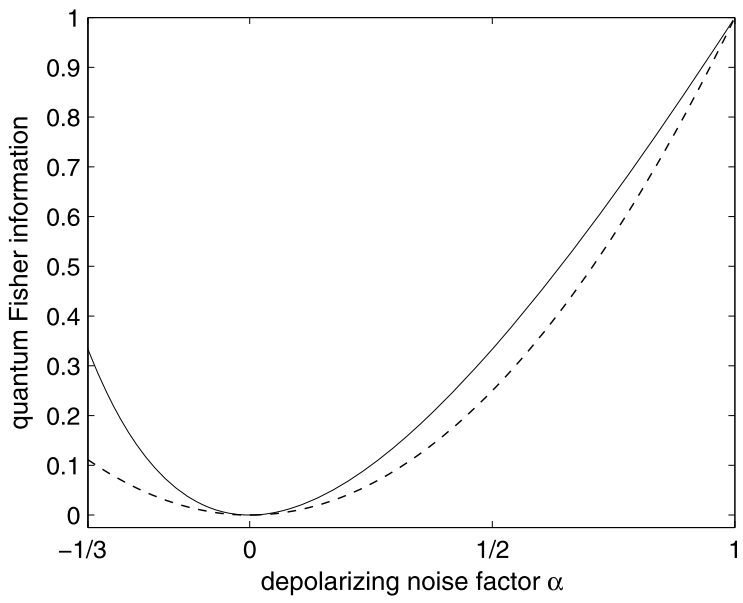

Fig. 2. Quantum Fisher information $F_{q}\left(\rho_{\xi} ; \xi\right)$ as a function of the depolarizing noise factor $\alpha \in[-1 / 3,1]$. Solid line: $F_{q}\left(\rho_{\xi} ; \xi\right)$ is from Eq. (29) with a noisy entangled qubit pair at $q=1 / 2$ where only one qubit in the pair experiences the unitary plus noise transformation via the setting of Fig. 1. Dashed line: $F_{q}\left(\rho_{\xi} ; \xi\right)$ is from Eq. (10), the maximal Fisher information accessible from a single independent noisy qubit.

$F_{q}\left(\rho_{\xi} ; \xi\right)=\frac{2 \alpha^{2}}{1+\alpha}$.

Fig. 2 compares the quantum Fisher information $F_{q}\left(\rho_{\xi} ; \xi\right)$ from the qubit pair of Eq. (29) to the maximal Fisher information accessible from a single qubit of Eq. (10).

It is visible in Fig. 2 that when there is no noise, at $\alpha=1$, the two-qubit setting of Fig. 1 and Eq. (29) performs no better than the single qubit of Eq. (10), with which it shares the quantum Fisher information $F_{q}\left(\rho_{\xi} ; \xi\right)=\alpha^{2}=1$. However, as soon as some noise comes into play, with $\alpha<1$, then the two-qubit setting of Fig. 1 and Eq. (29) becomes superior, with a larger quantum Fisher information in Eq. (29) compared to the maximal quantum Fisher information of the single qubit in Eq. (10). The gain or ratio of the two Fisher informations $2 /(1+\alpha)$ in Fig. 2 goes to 2 for a noise factor $|\alpha| \rightarrow 0$, and it goes to 3 as $\alpha \rightarrow-1 / 3$. Such superiority is specially remarkable, because, as expressed by Fig. 1, the second qubit of the pair never interacts with the $\xi$-dependent process to be estimated. Nevertheless, when it is entangled to the first qubit experiencing the $\xi$-dependent interaction, the qubit pair enables a net enhancement of the performance in estimation. This enhancement can be seen as a specifically quantum property, afforded by entanglement, with no classical analog, because classically when a probe does not interact with a process it cannot improve its estimation. Comparable benefit from entanglement with an inactive ancillary quantum system as in Fig. 1 has previously been observed for metrology, for instance in [23,14,29-32,13,33], yet in situations differing from the present one, either with no noise or with a performance metric differing from the quantum Fisher information employed here or another estimation task. The present study confirms and extends the situations where a specific benefit is accessible from quantum entanglement, especially for noisy quantum metrology.

\subsection{Two qubits transformed}

For comparison, it is also interesting to analyze the setting, depicted in Fig. 3, where the two qubits of the pair experience the unitary plus noise transformation, acting separately on each qubit. Such settings associating active entangled qubits uniformly in parallel are common for estimation and considered for instance in [11, $13,19,14,12,33]$. Here also, the characterization we develop is novel of the quantum Fisher information with depolarizing noise for such

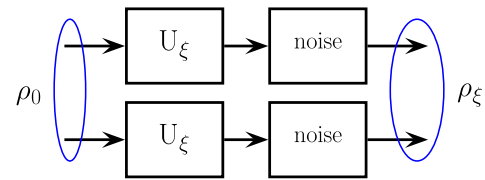

Fig. 3. A pair of qubits in a generally entangled state where the two qubits experience the unitary plus noise transformation.

a qubit pair. For the setting of Fig. 3, the resulting two-qubit noisy transformed state is now $\rho_{\xi}=\mathcal{T}_{\xi} \otimes \mathcal{T}_{\xi}\left(\rho_{0}\right)$.

When the input probe is in the general two-qubit state $\rho_{0}=$ $\left[\rho_{\text {bin }\left(j j^{\prime}\right) \text { bin }\left(k k^{\prime}\right)}\right]$, then from Eqs. (20)-(23) engaged in the tensor product, the two-qubit noisy transformed state $\rho_{\xi}=\mathcal{T}_{\xi} \otimes \mathcal{T}_{\xi}\left(\rho_{0}\right)$ in the canonical basis receives the matrix representation

$\rho \xi=\left[\begin{array}{cccc}d_{00} & \alpha e^{-i \xi}\left(b \rho_{01}+c \rho_{23}\right) & \alpha e^{-i \xi}\left(b \rho_{02}+c \rho_{13}\right) & \alpha^{2} e^{-i 2 \xi} \rho_{03} \\ \alpha e^{i \xi}\left(b \rho_{10}+c \rho_{32}\right) & d_{11} & \alpha^{2} \rho_{12} & \alpha e^{-i \xi}\left(c \rho_{02}+b \rho_{13}\right) \\ \alpha e^{i \xi}\left(b \rho_{20}+c \rho_{31}\right) & \alpha^{2} \rho_{21} & d_{22} & \alpha e^{-i \xi}\left(c \rho_{01}+b \rho_{23}\right) \\ \alpha^{2} e^{i 2 \xi} \rho_{30} & \alpha e^{i \xi}\left(c \rho_{20}+b \rho_{31}\right) & \alpha e^{i \xi}\left(c \rho_{10}+b \rho_{32}\right) & d_{33}\end{array}\right]$,

with the four diagonal terms

$d_{00}=b^{2} \rho_{00}+b c\left(\rho_{11}+\rho_{22}\right)+c^{2} \rho_{33}$,

$d_{11}=b^{2} \rho_{11}+b c\left(\rho_{00}+\rho_{33}\right)+c^{2} \rho_{22}$,

$d_{22}=b^{2} \rho_{22}+b c\left(\rho_{00}+\rho_{33}\right)+c^{2} \rho_{11}$,

$d_{33}=b^{2} \rho_{33}+b c\left(\rho_{11}+\rho_{22}\right)+c^{2} \rho_{00}$,

and the derivative

$\partial_{\xi} \rho_{\xi}=$

$\left[\begin{array}{cccc}0 & -i \alpha e^{-i \xi}\left(b \rho_{01}+c \rho_{23}\right) & -i \alpha e^{-i \xi}\left(b \rho_{02}+c \rho_{13}\right) & -i 2 \alpha^{2} e^{-i 2 \xi} \rho_{03} \\ i \alpha e^{i \xi}\left(b \rho_{10}+c \rho_{32}\right) & 0 & 0 & -i \alpha e^{-i \xi}\left(c \rho_{02}+b \rho_{13}\right) \\ i \alpha e^{i \xi}\left(b \rho_{20}+c \rho_{31}\right) & 0 & 0 & -i \alpha e^{-i \xi}\left(c \rho_{01}+b \rho_{23}\right) \\ i 2 \alpha^{2} e^{i 2 \xi} \rho_{30} & i \alpha e^{i \xi}\left(c \rho_{20}+b \rho_{31}\right) & i \alpha e^{i \xi}\left(c \rho_{10}+b \rho_{32}\right) & 0\end{array}\right]$.

With the input probe state $\rho_{0}$ taken under the form of Eqs. (27)-(28), the resulting two-qubit noisy transformed state $\rho_{\xi}=\mathcal{T}_{\xi} \otimes \mathcal{T}_{\xi}\left(\rho_{0}\right)$ from Eq. (30) is derived in Appendix B together with the ensuing quantum Fisher information $F_{q}\left(\rho_{\xi} ; \xi\right)$ of Eq. (1). The derivation of Appendix B shows a quantum Fisher information $F_{q}\left(\rho_{\xi} ; \xi\right)$ in Eq. (B.5) which is independent of the unknown parameter $\xi$, and which is again maximized by the Schmidt coefficient $q=1 / 2$, i.e. for a maximally entangled pure state for the input probe $\rho_{0}$ from Eq. (26). The corresponding maximal Fisher information achieved in Eq. (B.5) follows as

$F_{q}\left(\rho_{\xi} ; \xi\right)=\frac{8 \alpha^{4}}{1+\alpha^{2}}$

Fig. 4 compares the quantum Fisher information $F_{q}\left(\rho_{\xi} ; \xi\right)$ from the entangled qubit pair of Eq. (36) to the maximal Fisher information accessible from two separable qubits which is $2 \alpha^{2}$ after Eq. (10).

It is observed in Fig. 4 that when there is no noise, at $\alpha=1$, the two maximally entangled qubits of Fig. 3 are much more efficient for estimation since they lead to a Fisher information $F_{q}\left(\rho_{\xi} ; \xi\right)=4$ in Eq. (36) which is twice the maximal Fisher information $F_{q}\left(\rho_{\xi} ; \xi\right)=2 \alpha^{2}=2$ of two separable qubits. This is the quantum enhancement announced at the end of Section 3: with an $N$-qubit separable state the quantum Fisher information scales as $N$, while there is a possibility of scaling as $N^{2}$ with an $N$-qubit entangled state, as observed in Fig. 4 for $N=2$. However, as also announced at the end of Section 3, this quantum enhancement is significantly impaired by the presence of noise. In Fig. 4, when the level of noise increases as $\alpha$ decreases below 1 , the superiority of 


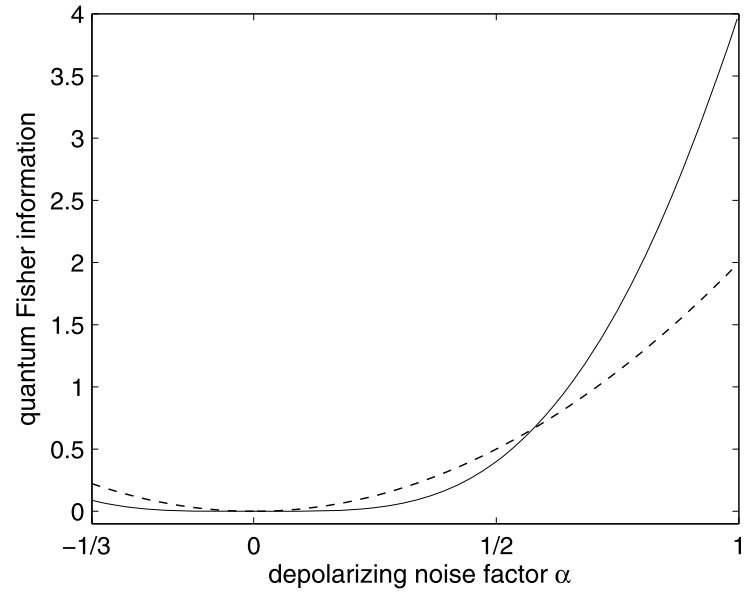

Fig. 4. Quantum Fisher information $F_{q}\left(\rho_{\xi} ; \xi\right)$ as a function of the depolarizing noise factor $\alpha \in[-1 / 3,1]$. Solid line: $F_{q}\left(\rho_{\xi} ; \xi\right)$ is from Eq. (36) with a noisy entangled qubit pair at $q=1 / 2$ where the two qubits in the pair experience the unitary plus noise transformation via the setting of Fig. 3. Dashed line: $F_{q}\left(\rho_{\xi} ; \xi\right)=2 \alpha^{2}$, the maximal Fisher information accessible from two separable noisy qubits after Eq. (10). A crossover occurs at $\alpha=\alpha_{1}=1 / \sqrt{3} \approx 0.577$.

the Fisher information $F_{q}\left(\rho_{\xi} ; \xi\right)$ of the entangled pair gradually diminishes. There is a crossover level, at $\alpha=\alpha_{1}=1 / \sqrt{3} \approx 0.577$ in Fig. 4. At higher noise levels, when $\alpha<\alpha_{1}$, the separable qubit pair shows a Fisher information $F_{q}\left(\rho_{\xi} ; \xi\right)=2 \alpha^{2}$ which becomes superior to that of the entangled pair in Eq. (36). This is a significant property. In the presence of noise, depending on the noise level, an entangled qubit pair may or may not be superior to a separable pair, in terms of quantum Fisher information for estimation. This provides further insight into the behavior of entanglement for estimation from noisy quantum systems. Entanglement as a quantum correlation makes the entangled pair in Fig. 3 react as a whole to the $\xi$-dependent unitary and to the noise, and in a way differing from the reaction of two independent separable qubits. Based on the quantitative behavior in Fig. 4, the qualitative interpretation is that, compared to the separable pair, at low noise, the entangled pair appears more responsive to the $\xi$-dependent unitary than to the noise, translating into a superior Fisher information. Conversely, at high noise, the entangled pair appears more sensitive to the noise than to the $\xi$-dependent unitary, translating into a poorer Fisher information. It is therefore important to realize the possibility of this crossover in the performance for estimation, and to be able, as accomplished here for a depolarizing noise on the qubit pair, to evaluate the level of noise where the crossover takes place, indicating when an entangled or a separable pair is preferable.

When the entangled qubit pair of Fig. 3 is strongly sensitive to the noise, at still higher noise level, its performance gets even poorer than that of the setting of Fig. 1 where only one qubit of the pair experiences the $\xi$-dependent unitary plus noise. This is made visible by Fig. 5, comparing the quantum Fisher information $F_{q}\left(\rho_{\xi} ; \xi\right)$ of Eq. (36) for the setting of Fig. 3 to that of Eq. (29) for the setting of Fig. 1. It is observed in Fig. 5 that another crossover occurs at $\alpha=\alpha_{2} \approx 0.455$, and for high noise levels where $\alpha<\alpha_{2}$ the setting of Fig. 3 with two active qubits has a poorer Fisher information than the setting of Fig. 1 with one active qubit.

In the setting of Fig. 3, each qubit undergoes a dual interaction including both a useful exposition to the $\xi$-dependent process under estimation and a harmful exposition to the noise. The net effect of such dual interaction is favorable at low noise, and detrimental at high noise. In this way, at high noise, it is preferable to limit such dual interaction and select the setting of Fig. 1 with only one qubit exposed to the unitary plus noise transformation, which turns out to be more efficient for estimation, as quantified here.

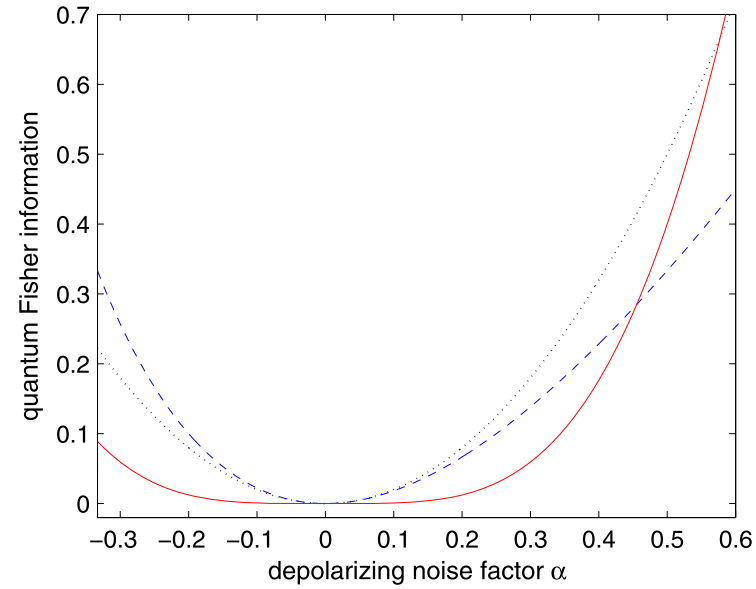

Fig. 5. Quantum Fisher information $F_{q}\left(\rho_{\xi} ; \xi\right)$ as a function of the depolarizing noise factor $\alpha$. Solid line: $F_{q}\left(\rho_{\xi} ; \xi\right)$ is from Eq. (36) with a noisy entangled qubit pair at $q=1 / 2$ where the two qubits in the pair experience the unitary plus noise transformation via the setting of Fig. 3. Dashed line: $F_{q}\left(\rho_{\xi} ; \xi\right)$ is from Eq. (29) with a noisy entangled qubit pair at $q=1 / 2$ where only one qubit in the pair experiences the unitary plus noise transformation via the setting of Fig. 1. Dotted line: $F_{q}\left(\rho_{\xi} ; \xi\right)=2 \alpha^{2}$, the maximal Fisher information accessible from two separable noisy qubits after Eq. (10).

\section{Optimized entanglement}

In terms of performance assessed by the quantum Fisher information $F_{q}\left(\rho_{\xi} ; \xi\right)$, the setting of Fig. 1 with only one qubit of the pair actively exposed to the unitary plus noise transformation and achieving $F_{q}\left(\rho_{\xi} ; \xi\right)$ of Eq. (29), is always superior to a single independent qubit exposed in the same way to the unitary plus noise transformation and achieving $F_{q}\left(\rho_{\xi} ; \xi\right)=\alpha^{2}$ of Eq. (10), as visible in Fig. 2. In addition, two independent qubits achieving $F_{q}\left(\rho_{\xi} ; \xi\right)=2 \alpha^{2}$ are always superior to the setting with one active qubit of Fig. 1 achieving $F_{q}\left(\rho_{\xi} ; \xi\right)$ of Eq. (29). Meanwhile, two active entangled qubits as in Fig. 3 achieving $F_{q}\left(\rho_{\xi} ; \xi\right)$ of Eq. (36) are superior to two independent qubits only at low noise, as visible in Fig. 4. As we are going to see, there is however some room for further improving the performance of the two entangled qubits of Fig. 3 over that of the two independent qubits. This is possible by entangling the input probe in a different way, as we now examine.

Based on Section 3, for estimation of the phase $\xi$ in Eq. (11) from one-qubit independent states, the maximal quantum Fisher information $F_{q}^{\max }=\alpha^{2}$ of Eq. (10) can be reached by a one-qubit input probe prepared in the pure state $|+\rangle=(|0\rangle+|1\rangle) / \sqrt{2}$. Consequently, with a pair of two independent qubits, twice as much quantum Fisher information $F_{q}\left(\rho_{\xi} ; \xi\right)=2 \alpha^{2}$ is obtained with the two-qubit separable input probe $|+\rangle^{\otimes 2}$. These represent the conditions of maximal Fisher information accessible from two independent qubits, and this remains true for any level or factor $\alpha$ of the depolarizing noise.

With no noise at $\alpha=1$, when a single qubit enables to reach the maximal Fisher information $F_{q}^{\max }=1$, it is known that $N$ entangled qubits can at most reach the maximal Fisher information $N^{2} F_{q}^{\max }=N^{2}[12,11]$. Therefore, with no noise at $\alpha=1$, the twoqubit input probe $(|00\rangle+|11\rangle) / \sqrt{2}$ at $q=1 / 2$ in Eq. (26), effectively achieves this maximal Fisher information $F_{q}\left(\rho_{\xi} ; \xi\right)=N^{2}=4$ as seen in Fig. 4, and no other two-qubit entangled probe can do better.

When some noise starts to appear, with the noise factor $\alpha$ decreasing below 1, one can expect that at low noise ( $\alpha$ close to 1 ), the input probe $(|00\rangle+|11\rangle) / \sqrt{2}$ will remain quasi-optimal to elicit the maximum of the quantum Fisher information $F_{q}\left(\rho_{\xi} ; \xi\right)$. Beyond, for an arbitrary noise factor $\alpha<1$, the determination of 


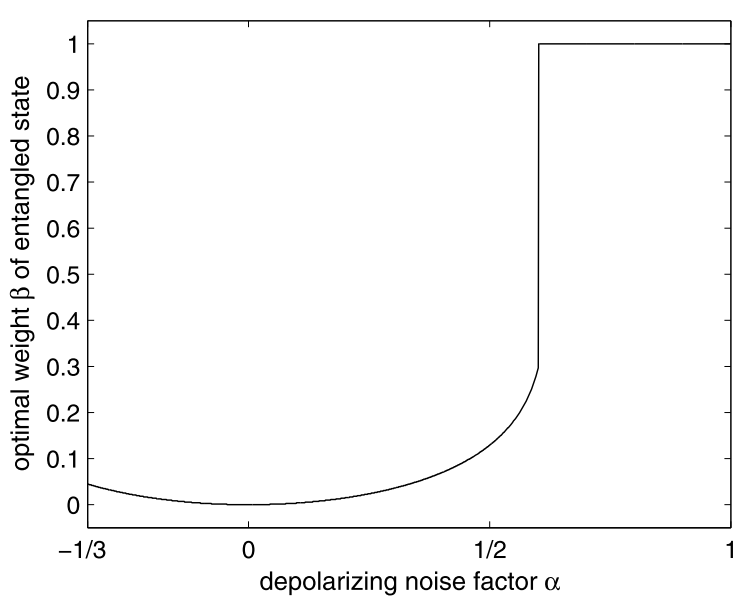

Fig. 6. Optimal weight $\beta$ of the entangled state $(|00\rangle+|11\rangle) / \sqrt{2}$ in the combination of Eq. (37) to achieve the maximum of the quantum Fisher information $F_{q}\left(\rho_{\xi} ; \xi\right)$ of Eq. (1), as a function of the depolarizing noise factor $\alpha$.

the optimal input probe maximizing the quantum Fisher information $F_{q}\left(\rho_{\xi} ; \xi\right)$ is accomplished for a one-qubit probe in Section 3, but it becomes a more complicated task for a two-qubit probe. From Section 2, it is generally known that the optimal input probe maximizing the quantum Fisher information is necessarily a pure quantum state $\left|\psi_{0}\right\rangle$. For a two-qubit probe, a pure quantum state $\left|\psi_{0}\right\rangle$ is generally parametrized by 7 real numbers which, via $\rho_{0}=\left|\psi_{0}\right\rangle\left\langle\psi_{0}\right|$, will control the transformed state $\rho_{\xi}$. An eigendecomposition of this $\rho_{\xi}$ has then to be performed in order to characterize the quantum Fisher information $F_{q}\left(\rho_{\xi} ; \xi\right)$ of Eq. (1) that need be maximized according to these 7 real variables. Such multidimensional constrained optimization is usually difficult to accomplish [17,19]. As an alternative, to obtain further insight on beneficial aspects of entanglement for estimation, one can address this optimization in a restricted class of two-qubit pure states. Since the probe $|+\rangle^{\otimes 2}$ is optimal for two independent qubits at any noise level $\alpha$, and the probe $(|00\rangle+|11\rangle) / \sqrt{2}$ is optimal for two entangled qubits with no noise at $\alpha=1$, at an arbitrary noise level $\alpha$ one can look for an optimal (pure) probe as the weighted combination

$\left|\psi_{0}\right\rangle=\beta \frac{|00\rangle+|11\rangle}{\sqrt{2}}+\beta^{\prime}|+\rangle^{\otimes 2}$.

For a valid (normalized) pure state $\left|\psi_{0}\right\rangle$, the two weights in Eq. (37) have to satisfy $\beta^{\prime}=-\beta / \sqrt{2} \pm\left[1-(\beta / \sqrt{2})^{2}\right]^{1 / 2}$ and are chosen real so that Eq. (37) realize a parametrization with one independent (real) parameter; in addition $\beta \in[-\sqrt{2}, \sqrt{2}]$ is required. It is then interesting to look for the optimal weight $\beta$ providing an input probe $\left|\psi_{0}\right\rangle$ in Eq. (37) achieving the maximum of the quantum Fisher information $F_{q}\left(\rho_{\xi} ; \xi\right)$ in Eq. (1). This can be accomplished numerically, by computing for any given $\beta$, via $\rho_{0}=\left|\psi_{0}\right\rangle\left\langle\psi_{0}\right|$, the transformed state $\rho_{\xi}$ of Eq. (30), then the eigendecomposition of this $4 \times 4$ matrix $\rho_{\xi}$, to be used with $\partial_{\xi} \rho_{\xi}$ of Eq. (35) so as to evaluate $F_{q}\left(\rho_{\xi} ; \xi\right)$ of Eq. (1). The resulting optimal weight $\beta$ is presented in Fig. 6, where from symmetry of Eq. (37), for any $\beta$ optimal, $-\beta$ offers an equivalent optimal solution.

The optimal value $\beta_{\text {opt }}$ of the weight $\beta$ plotted in Fig. 6 shows a sharp transition at a critical value $\alpha_{c} \approx 0.601$ of the depolarizing noise factor $\alpha$. At low noise, when $\alpha>\alpha_{c}$, the optimal weight in Fig. 6 is uniformly $\beta_{\text {opt }}=1$ independent of $\alpha$, indicating that in this range of noise, when the pure input probe is formed as the combination of Eq. (37), then it is the maximally entangled probe $\left|\psi_{0}\right\rangle=(|00\rangle+|11\rangle) / \sqrt{2}$ that always maximizes the quantum Fisher information $F_{q}\left(\rho_{\xi} ; \xi\right)$ at a level given by Eq. (36). On the contrary,

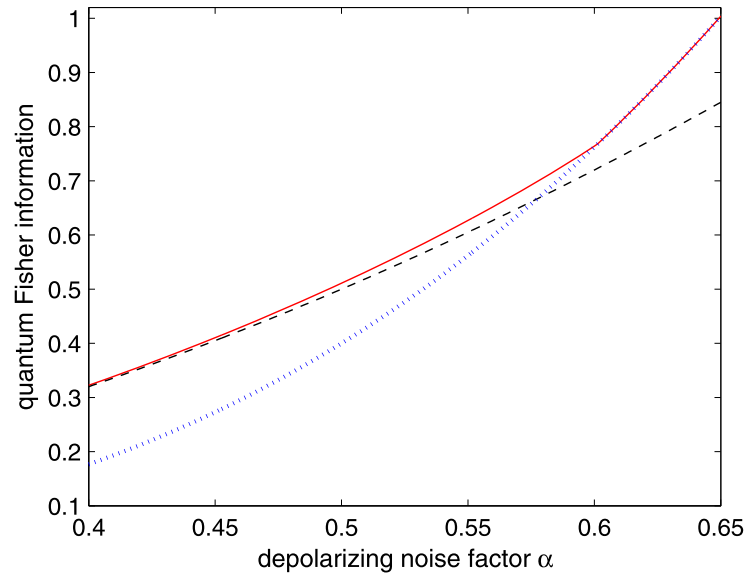

Fig. 7. Solid line: maximum of the quantum Fisher information $F_{q}\left(\rho_{\xi} ; \xi\right)$ in Eq. (1) achieved by the input probe $\left|\psi_{0}\right\rangle$ of Eq. (37) with the optimal weight $\beta$ of the entangled state $(|00\rangle+|11\rangle) / \sqrt{2}$ given in Fig. 6. Dotted and dashed lines are the same quantum Fisher informations $F_{q}\left(\rho_{\xi} ; \xi\right)$ as in Fig. 4, with here in dotted line $F_{q}\left(\rho_{\xi} ; \xi\right)$ from Eq. (36) of the maximally entangled input probe $(|00\rangle+|11\rangle) / \sqrt{2}$, and in dashed line $F_{q}\left(\rho_{\xi} ; \xi\right)=2 \alpha^{2}$ of the separable input probe $|+\rangle^{\otimes 2}$.

at high noise when $\alpha<\alpha_{c}$, the optimal weight $\beta_{\text {opt }}$ in Fig. 6 becomes $\alpha$-dependent and steadily decreases below 1 . This indicates that the optimal input probe $\left|\psi_{0}\right\rangle$ in Eq. (37) now combines a part of the maximally entangled state $(|00\rangle+|11\rangle) / \sqrt{2}$ and a part of the separable state $|+\rangle^{\otimes 2}$, matched to the noise level $\alpha$. The optimal probe $\left|\psi_{0}\right\rangle$ is in this respect a partially entangled state and no longer a maximally entangled state. Only asymptotically at very large noise when $|\alpha| \rightarrow 0$ does the optimal weight $\beta_{\text {opt }} \rightarrow 0$ in Fig. 6, identifying an optimal input probe $\left|\psi_{0}\right\rangle$ in Eq. (37) that progressively ceases to be entangled to tend to the separable state $|+\rangle^{\otimes 2}$, associated with the performance $F_{q}\left(\rho_{\xi} ; \xi\right) \rightarrow 2 \alpha^{2}$ that also vanishes at $\alpha \rightarrow 0$, indicating a separable probe that although optimal becomes also inoperative for estimation. A comparable numerical optimization of an input probe in a task of frequency estimation was also performed in [16], yet with another performance metric, and an optimum was also found at a partially entangled probe but no sharp transition at a critical noise level as in Fig. 6.

The maximal quantum Fisher information $F_{q}\left(\rho_{\xi} ; \xi\right)$ achieved by the input probe $\left|\psi_{0}\right\rangle$ of Eq. (37) at $\beta_{\text {opt }}$ is shown in Fig. 7, as a function of the depolarizing noise factor $\alpha$. As explained above, at $\alpha>\alpha_{c}$ then $\beta_{\text {opt }}=1$ and the maximal Fisher information is $F_{q}\left(\rho_{\xi} ; \xi\right)$ of Eq. (36), meanwhile as $|\alpha| \rightarrow 0$ then $\beta_{\text {opt }} \rightarrow 0$ and the maximal $F_{q}\left(\rho_{\xi} ; \xi\right)$ tends to $2 \alpha^{2}$. In addition, at $\alpha<\alpha_{c}$, the maximal $F_{q}\left(\rho_{\xi} ; \xi\right)$ of the partially entangled optimal probe at $\beta_{\text {opt }}$, is always above, simultaneously, $F_{q}\left(\rho_{\xi} ; \xi\right)$ of Eq. (36) of the maximally entangled probe $(|00\rangle+|11\rangle) / \sqrt{2}$ and $F_{q}\left(\rho_{\xi} ; \xi\right)=2 \alpha^{2}$ of the separable probe $|+\rangle^{\otimes 2}$. However, as revealed by Fig. 7, the maximal $F_{q}\left(\rho_{\xi} ; \xi\right)$ at any $\beta_{\text {opt }}$ never departs much from its two limiting values at $\beta_{\text {opt }}=1$ or at $\beta_{\text {opt }} \rightarrow 0$, whichever of the two is larger. The maximal departure is at the crossover $\alpha=\alpha_{1}=1 / \sqrt{3} \approx 0.577$ introduced while analyzing Fig. 4. At $\alpha=\alpha_{1}$ in Fig. 7, with $\beta_{\text {opt }} \approx$ 0.228 the maximal $F_{q}\left(\rho_{\xi} ; \xi\right)$ is $\approx 0.698$ while the common value of the two limiting Fisher informations is $2 / 3$.

We know from Section 3 that for a separable qubit pair the maximum of the quantum Fisher information is $F_{q}\left(\rho_{\xi} ; \xi\right)=2 \alpha^{2}$, achievable by the separable probe $|+\rangle^{\otimes 2}$. The analysis of the performance of Eq. (37) as in Fig. 7, shows that there always exists an entangled probe in Eq. (37), characterized by a nonzero optimal weight $\beta$ in Fig. 6, achieving a higher quantum Fisher information than the separable probe. And this superiority of a (possibly partially) entangled probe over a separable probe is true for any nonzero noise factor $\alpha$. In this respect, for estimation with depo- 
larizing noise, entanglement is always capable of enhancing the performance over that of the optimal separable pair.

\section{Conclusion}

In this report, various benefits have been quantified that can be obtained from entanglement for quantum parameter estimation especially in the presence of noise or decoherence. The performance in estimation is assessed by the quantum Fisher information, which is a fundamental metric fixing the ultimate best performance. In general, when an input probe state experiences a $\xi$-dependent transformation, then it was shown in Section 2 that the quantum Fisher information for estimating $\xi$ is always maximized by a pure input probe. We then turned to a generic class of estimation task, consisting in estimating the phase $\xi$ of a unitary transformation on a qubit, in the presence of depolarizing noise. As a reference, when one operates with independent qubits prepared in a separable state, the optimal input probe was characterized in Section 3 together with the maximal quantum Fisher information it achieves as a function of the level of noise. When only one qubit interacts with the $\xi$-dependent unitary plus noise, it was shown in Section 4.1 that a net enhancement over the one-qubit optimal probe for the quantum Fisher information $F_{q}\left(\rho_{\xi} ; \xi\right)$ can always be obtained by maximally entangling such an active qubit with an inactive ancillary qubit that never interacts with the $\xi$-dependent process (i.e. the setting of Fig. 1). This is a purely quantum benefit, with no classical analog, illustrating how the specific quantum correlation realized by entanglement can result in an improved performance in noisy conditions of estimation. With two active qubits as in Fig. 3, it was shown in Section 4.2 that a maximally entangled probe is more efficient than the optimal separable probe provided the noise is not too high. However, in Section 5 it was shown that, even at high noise, a partially entangled probe always exists that outperforms the optimal separable probe. Yet for practical purposes the margin of improvement may be small, and it is therefore useful to evaluate the crossover level $\alpha_{1}$ of the noise, as done in Fig. 4, indicating when, among these two practically simpler configurations, the maximally entangled or the separable two-qubit probe is preferable.

The present approach quantifying benefit from entanglement for estimation in the presence of noise can be extended in several directions. It can be extended to other types of quantum noise relevant to the qubit. The depolarizing noise investigated here represents an important reference for the qubit, but the approach implemented here can in principle be reproduced for any other noise on the qubit. A specific noise process will be specified by its Kraus operators $\Lambda_{\ell}$ in Eq. (8), and it is known that a maximum of four $\Lambda_{\ell}$ are sufficient to represent any arbitrary noise on the qubit. From there the same steps followed here can be reproduced to obtain the noisy state $\rho_{\xi}$ of the qubit pair similar to Eqs. (A.1) or (B.1), and perform the eigendecomposition of $\rho_{\xi}$ giving access to the quantum Fisher information $F_{q}\left(\rho_{\xi} ; \xi\right)$ of Eq. (1) to be studied as a function of the noise properties in various configurations of entanglement of the pair. The depolarizing noise with its isotropic action in Bloch representation enabled a rather thorough analytic completion of the approach, as demonstrated here, to provide useful insight. There nevertheless exist more involved noises also relevant to the qubit, such as amplitude damping or generalized amplitude damping or squeezed amplitude damping noises [25,28,34]. Their mode of action is however more involved and controlled by a larger number of parameters, which may complicate the analytical treatment. Extension of the approach to such noises is in principle feasible, and this represents an interesting perspective open for further exploration. For estimation on such noisy qubits, it may be expected that some benefit from entanglement as reported here can persist, as essentially stemming from the very specific and unparalleled coupling formed by entanglement, and not critically affected by varying the type of noise. This however remains to be investigated explicitly.

Extension of the present work can also be envisaged to multiparametric estimation, or to quantum systems of dimension larger than the dimension two of the qubit. However, here also the analysis is likely to be more difficult to handle analytically. It is therefore useful to identify as guidelines, situations as here that can be treated analytically so as to provide more insight meaningful to quantum information with entanglement and noise. Quantum noise or decoherence is an essential factor limiting the operation of quantum information technologies. It is therefore relevant to further analyze and optimize quantum information processes in the presence of noise, with a base at the level of the qubit which is a fundamental system for quantum information. Also, as a specifically quantum and unparalleled property, entanglement could be further explored for its beneficial role for quantum information and quantum signal processing in the presence of noise, as for instance for application to quantum state discrimination or detection $[35,14,34]$ or for the exploitation of nonlocal quantum correlations [36-38] for noisy quantum information.

\section{Appendix A. One qubit transformed}

For the setting of Fig. 1, with the input probe state $\rho_{0}$ taken under the form of Eqs. (27)-(28), the resulting two-qubit noisy transformed state $\rho_{\xi}=\mathcal{T}_{\xi} \otimes \mathcal{I}\left(\rho_{0}\right)$ from Eq. (24) follows as

$$
\rho_{\xi}=\left[\begin{array}{cccc}
(1-q) b & 0 & 0 & \sqrt{(1-q) q} \alpha e^{-i \xi} \\
0 & q c & 0 & 0 \\
0 & 0 & (1-q) c & 0 \\
\sqrt{(1-q) q} \alpha e^{i \xi} & 0 & 0 & q b
\end{array}\right],
$$

and its derivative from Eq. (25),

$$
\partial_{\xi} \rho_{\xi}=\left[\begin{array}{cccc}
0 & 0 & 0 & -\sqrt{(1-q) q} i \alpha e^{-i \xi} \\
0 & 0 & 0 & 0 \\
0 & 0 & 0 & 0 \\
\sqrt{(1-q) q} i \alpha e^{i \xi} & 0 & 0 & 0
\end{array}\right] .
$$

For the eigendecomposition of $\rho_{\xi}$, the form of Eq. (A.1) shows that in $\mathcal{H}_{2}^{\otimes 2}$ the state vector $|01\rangle=[0,1,0,0]^{\top}$ is eigenvector of $\rho_{\xi}$ with eigenvalue $q c$, while the state vector $|10\rangle=[0,0,1,0]^{\top}$ is eigenvector of $\rho_{\xi}$ with eigenvalue $(1-q) c$. Since $\rho_{\xi}$ is Hermitian with four mutually orthogonal eigenvectors, the two remaining eigenvectors of $\rho_{\xi}$ are to be found in the two-dimensional subspace spanned by $(|00\rangle,|11\rangle)$, where they can be searched under the form

$|\lambda\rangle=a_{0}|00\rangle+a_{1}|11\rangle$.

From Eq. (A.1), the transformation by $\rho_{\xi}$ of such a $|\lambda\rangle$ occurs in the plane $(|00\rangle,|11\rangle)$ and can be described by the matrix operation

$\left[\begin{array}{cc}(1-q) b & \sqrt{(1-q) q} \alpha e^{-i \xi} \\ \sqrt{(1-q) q} \alpha e^{i \xi} & q b\end{array}\right]\left[\begin{array}{l}a_{0} \\ a_{1}\end{array}\right]=\rho_{\xi}^{(2)}|\lambda\rangle$,

with the $2 \times 2$ matrix $\rho_{\xi}^{(2)}$ defining the operation of the operator $\rho_{\xi}$ restricted to the plane $(|00\rangle,|11\rangle)$. We are faced with an eigendecomposition restricted to the plane $(|00\rangle,|11\rangle)$, with a characteristic equation $\operatorname{det}\left(\rho_{\xi}^{(2)}-\lambda \mathrm{I}_{2}\right)=0$ equivalent to $\lambda^{2}-$ $b \lambda+(1-q) q\left(b^{2}-\alpha^{2}\right)=0$. This second-degree equation in $\lambda$ has the discriminant $\Delta=b^{2}-4(1-q) q\left(b^{2}-\alpha^{2}\right) \geq 0$, and generally two roots providing the two (real) eigenvalues that we seek as $\lambda_{ \pm}=(b \pm \sqrt{\Delta}) / 2$. Especially useful to us in the sequel is the sum $\lambda_{+}+\lambda_{-}=b=(1+\alpha) / 2$. 
Now that the two eigenvalues $\lambda_{ \pm}$are expressed, the two corresponding eigenvectors $\left|\lambda_{ \pm}\right\rangle$readily follow by solving the two linear systems $\rho_{\xi}^{(2)}\left|\lambda_{ \pm}\right\rangle=\lambda_{ \pm}\left|\lambda_{ \pm}\right\rangle$. We do not write explicitly the resulting expressions, noting that for the sequel it is enough to use the characterization $\left|\lambda_{+}\right\rangle=a_{0}|00\rangle+a_{1} e^{i \xi}|11\rangle$ and $\left|\lambda_{-}\right\rangle=a_{1} e^{-i \xi}|00\rangle-$ $a_{0}|11\rangle$, with now $a_{0}$ and $a_{1}$ real, for two orthonormal eigenstates $\left|\lambda_{ \pm}\right\rangle$lying in the plane $(|00\rangle,|11\rangle)$.

For our objective of computing the quantum Fisher information $F_{q}\left(\rho_{\xi} ; \xi\right)$ of Eq. (1), due to the form of $\partial_{\xi} \rho_{\xi}$ in Eq. (A.2), it is clear that only the two eigenvectors $\left|\lambda_{ \pm}\right\rangle$lying in the plane $(|00\rangle,|11\rangle)$ can contribute a non-vanishing scalar $\left\langle\lambda_{m}\left|\partial_{\xi} \rho_{\xi}\right| \lambda_{n}\right\rangle$ in Eq. (1). Moreover, with the above form determined for $\left|\lambda_{ \pm}\right\rangle$, it follows that each of the two rectangular terms $\left\langle\lambda_{+}\left|\partial_{\xi} \rho_{\xi}\right| \lambda_{+}\right\rangle$ and $\left\langle\lambda_{-}\left|\partial_{\xi} \rho_{\xi}\right| \lambda_{-}\right\rangle$is identically zero. Only the two diagonal terms $\left\langle\lambda_{+}\left|\partial_{\xi} \rho_{\xi}\right| \lambda_{-}\right\rangle=\left\langle\lambda_{-}\left|\partial_{\xi} \rho_{\xi}\right| \lambda_{+}\right\rangle^{*}$ do not vanish, but yield

$\left\langle\lambda_{+}\left|\partial_{\xi} \rho_{\xi}\right| \lambda_{-}\right\rangle=\sqrt{(1-q) q} i \alpha e^{-i \xi}$.

The quantum Fisher information $F_{q}\left(\rho_{\xi} ; \xi\right)$ of Eq. (1) finally evaluates to

$F_{q}\left(\rho_{\xi} ; \xi\right)=4(1-q) q \frac{2 \alpha^{2}}{1+\alpha}$.

\section{Appendix B. Two qubits transformed}

For the setting of Fig. 3 , with the input probe state $\rho_{0}$ taken under the form of Eqs. (27)-(28), the resulting two-qubit noisy transformed state $\rho_{\xi}=\mathcal{T}_{\xi} \otimes \mathcal{T}_{\xi}\left(\rho_{0}\right)$ from Eq. (30) follows as

$\rho_{\xi}=\left[\begin{array}{cccc}(1-q) b^{2}+q c^{2} & 0 & 0 & \sqrt{(1-q) q} \alpha^{2} e^{-i 2 \xi} \\ 0 & b c & 0 & 0 \\ 0 & 0 & b c & 0 \\ \sqrt{(1-q) q} \alpha^{2} e^{i 2 \xi} & 0 & 0 & (1-q) c^{2}+q b^{2}\end{array}\right]$,

and its derivative from Eq. (35),

$\partial_{\xi} \rho_{\xi}=\left[\begin{array}{cccc}0 & 0 & 0 & -\sqrt{(1-q) q} i 2 \alpha e^{-i 2 \xi} \\ 0 & 0 & 0 & 0 \\ 0 & 0 & 0 & 0 \\ \sqrt{(1-q) q} i 2 \alpha e^{i 2 \xi} & 0 & 0 & 0\end{array}\right]$.

The eigendecomposition of $\rho_{\xi}$ from Eq. (B.1) follows the same steps as that of $\rho_{\xi}$ from Eq. (A.1). Both $|01\rangle=[0,1,0,0]^{\top}$ and $|10\rangle=[0,0,1,0]^{\top}$ are eigenvector of $\rho_{\xi}$ in Eq. (B.1) with same eigenvalue $b c=\left(1-\alpha^{2}\right) / 4$. The other two eigenvectors are in the plane $(|00\rangle,|11\rangle)$, and solutions to the two-dimensional eigendecomposition of the matrix

$\rho_{\xi}^{(2)}=\left[\begin{array}{cc}(1-q) b^{2}+q c^{2} & \sqrt{(1-q) q} \alpha^{2} e^{-i 2 \xi} \\ \sqrt{(1-q) q} \alpha^{2} e^{i 2 \xi} & (1-q) c^{2}+q b^{2}\end{array}\right]$

comparable to Eq. (A.4). Similarly, the resulting degree-two characteristic equation $\operatorname{det}\left(\rho_{\xi}^{(2)}-\lambda \mathrm{I}_{2}\right)=0$ has two real roots $\lambda_{ \pm}$summing now to $\lambda_{+}+\lambda_{-}=b^{2}+c^{2}=\left(1+\alpha^{2}\right) / 2$. Due to the form of Eq. (B.2) for $\partial_{\xi} \rho_{\xi}$, only the two associated eigenvectors $\left|\lambda_{ \pm}\right\rangle$lying in the plane $(|00\rangle,|11\rangle)$ contribute to the quantum Fisher information $F_{q}\left(\rho_{\xi} ; \xi\right)$ of Eq. (1), moreover only through the two diagonal terms

$\left\langle\lambda_{+}\left|\partial_{\xi} \rho_{\xi}\right| \lambda_{-}\right\rangle=\left\langle\lambda_{-}\left|\partial_{\xi} \rho_{\xi}\right| \lambda_{+}\right\rangle^{*}=\sqrt{(1-q) q} i 2 \alpha^{2} e^{-i 2 \xi}$,

while each of the two rectangular terms $\left\langle\lambda_{+}\left|\partial_{\xi} \rho_{\xi}\right| \lambda_{+}\right\rangle$and $\left\langle\lambda_{-}\left|\partial_{\xi} \rho_{\xi}\right| \lambda_{-}\right\rangle$vanishes.

Finally, the quantum Fisher information $F_{q}\left(\rho_{\xi} ; \xi\right)$ of Eq. (1) evaluates to
$F_{q}\left(\rho_{\xi} ; \xi\right)=4(1-q) q \frac{8 \alpha^{4}}{1+\alpha^{2}}$

\section{References}

[1] S.M. Kay, Fundamentals of Statistical Signal Processing: Estimation Theory, Prentice Hall, Englewood Cliffs, 1993.

[2] C.W. Helstrom, The minimum variance of estimates in quantum signal detection, IEEE Trans. Inf. Theory 14 (1968) 234-242.

[3] S.L. Braunstein, C.M. Caves, Statistical distance and the geometry of quantum states, Phys. Rev. Lett. 72 (1994) 3439-3443.

[4] A. Fujiwara, H. Nagaoka, Quantum Fisher metric and estimation for pure state models, Phys. Lett. A 201 (1995) 119-124

[5] O.E. Barndorff-Nielsen, R.D. Gill, Fisher information in quantum statistics, J. Phys. A 33 (2000) 4481-4490.

[6] M.A. Armen, J.K. Au, J.K. Stockton, A.C. Doherty, H. Mabuchi, Adaptive homodyne measurement of optical phase, Phys. Rev. Lett. 89 (2002) 133602.

[7] A. Fujiwara, Strong consistency and asymptotic efficiency for adaptive quantum estimation problems, J. Phys. A 39 (2006) 12489-12504.

[8] D. Brivio, S. Cialdi, S. Vezzoli, B.T. Gebrehiwot, M.G. Genoni, S. Olivares, M.G.A. Paris, Experimental estimation of one-parameter qubit gates in the presence of phase diffusion, Phys. Rev. A 81 (2010) 012305.

[9] E. Tesio, S. Olivares, M.G.A. Paris, Optimized qubit phase estimation in noisy quantum channels, Int. J. Quantum Inf. 9 (2011) 379-387.

[10] R. Okamoto, M. Iefuji, S. Oyama, K. Yamagata, H. Imai, A. Fujiwara, S. Takeuchi, Experimental demonstration of adaptive quantum state estimation, Phys. Rev. Lett. 109 (2012) 130404.

[11] Z. Ji, G. Wang, R. Duan, Y. Feng, M. Ying, Parameter estimation of quantum channels, IEEE Trans. Inf. Theory 54 (2008) 5172-5185.

[12] V. Giovannetti, S. Lloyd, L. Maccone, Quantum metrology, Phys. Rev. Lett. 96 (2006) 010401.

[13] V. Giovannetti, S. Lloyd, L. Maccone, Advances in quantum metrology, Nat. Photonics 5 (2011) 222-229.

[14] G.M. D’Ariano, P. Lo Presti, M.G.A. Paris, Using entanglement improves the precision of quantum measurements, Phys. Rev. Lett. 87 (2001) 270404.

[15] V. Giovannetti, S. Lloyd, L. Maccone, Quantum-enhanced measurements: beating the standard quantum limit, Science 306 (2004) 1330-1336.

[16] S.F. Huelga, C. Macchiavello, T. Pellizzari, A.K. Ekert, M.B. Plenio, J.I. Cirac, Improvement of frequency standards with quantum entanglement, Phys. Rev. Lett. 79 (1997) 3865-3868

[17] B.M. Escher, R.L. de Matos Filho, L. Davidovich, General framework for estimating the ultimate precision limit in noisy quantum-enhanced metrology, Nat. Phys. 7 (2011) 406-411.

[18] D. Xie, A.M. Wang, Quantum metrology in correlated environments, Phys. Lett. A 378 (2014) 2079-2084.

[19] R. Demkowicz-Dobrzański, J. Kolodyński, M. Gută, The elusive Heisenberg limit in quantum-enhanced metrology, Nat. Commun. 3 (2012) 1063.

[20] A.S. Holevo, Probabilistic and Statistical Aspects of Quantum Theory, NorthHolland, Amsterdam, 1982.

[21] M.G.A. Paris, Quantum estimation for quantum technology, Int. J. Quantum Inf. 7 (2009) 125-137.

[22] F. Chapeau-Blondeau, Optimized probing states for qubit phase estimation with general quantum noise, Phys. Rev. A 91 (2015) 052310.

[23] A. Fujiwara, Quantum channel identification problem, Phys. Rev. A 63 (2001) 042304

[24] S. Alipour, A.T. Rezakhani, Extended convexity of quantum Fisher information in quantum metrology, Phys. Rev. A 91 (2015) 042104

[25] M.A. Nielsen, I.L. Chuang, Quantum Computation and Quantum Information, Cambridge University Press, Cambridge, 2000.

[26] F. Chapeau-Blondeau, Optimizing qubit phase estimation, Phys. Rev. A 94 (2016) 022334.

[27] F. Chapeau-Blondeau, Quantum state discrimination and enhancement by noise, Phys. Lett. A 378 (2014) 2128-2136.

[28] M.M. Wilde, Quantum Information Theory, Cambridge University Press, Cambridge, 2013.

[29] D.G. Fischer, H. Mack, M.A. Cirone, M. Freyberger, Enhanced estimation of a noisy quantum channel using entanglement, Phys. Rev. A 64 (2001) 022309.

[30] F. De Martini, A. Mazzei, M. Ricci, G.M. D’Ariano, Exploiting quantum parallelism of entanglement for a complete experimental quantum characterization of a single-qubit device, Phys. Rev. A 67 (2003) 062307.

[31] G.M. D’Ariano, P. Lo Presti, Imprinting complete information about a quantum channel on its output state, Phys. Rev. Lett. 91 (2003) 047902.

[32] M.A. Ballester, Estimation of unitary quantum operations, Phys. Rev. A 69 (2004) 022303.

[33] A. Smirne, J. Kołodyński, S.F. Huelga, R. Demkowicz-Dobrzański, Ultimate precision limits for noisy frequency estimation, Phys. Rev. Lett. 116 (2015) 120801.

[34] F. Chapeau-Blondeau, Optimization of quantum states for signaling across an arbitrary qubit noise channel with minimum-error detection, IEEE Trans. Inf. Theory 61 (2015) 4500-4510. 
[35] A.M. Childs, J. Preskill, J. Renes, Quantum information and precision measurement, J. Mod. Opt. 47 (2000) 155-176.

[36] F.A. Bovino, G. Castagnoli, A. Cabello, A. Lamas-Linares, Experimental noiseresistant Bell-inequality violations for polarization-entangled photons, Phys. Rev. A 73 (2006) 062110.
[37] F. Chapeau-Blondeau, Tsallis entropy for assessing quantum correlation with Bell-type inequalities in EPR experiment, Physica A 414 (2014) 204-215.

[38] H.C. Nguyen, T. Vu, Nonseparability and steerability of two-qubit states from the geometry of steering outcomes, Phys. Rev. A 94 (2016) 012114. 